\title{
Adaptation of hand tracking to rotated visual coordinates
}

\author{
C. PRABLANC, A. TZAVARAS, and M. JEANNEROD \\ Laboratoire de Neuropsychologie Expérimentale, U 94 INSERM, 69500 Bron, France
}

\begin{abstract}
A newly developed technique, allowing continuous recording of hand movements during the tracing of lines on a horizontal writing surface, was used to measure aftereffects induced by the exposure of subjects to a rotation $(20 \mathrm{deg})$ of the visual-motor feedback from their own hands. All subjects exhibited large aftereffects following this exposure when tested on tracing lines at given orientations in an open-loop situation. If the exposure was restricted to the tracing of lines of particular orientations, the aftereffect was nevertheless observed in all the orientations subsequently tested. These results tend to demonstrate a generalization of the adaptive effect and to support the hypothesis of a "recalibration" mechanism for the observed adaptation.
\end{abstract}

Rotation of visual coordinates around the visual axis (e.g., by viewing the environment through Dove prisms) makes lines that are physically vertical or horizontal appear tilted. Prolonged inspection of these tilted lines produces a perceptual "adaptation" first described by Gibson (1937). When the visual coordinates are set back in concordance with the physical coordinates (e.g., by removing the prisms), the subject sees as "vertical" lines that are physically tilted in the direction to which he has adapted (provided the tilt of exposure was less than $45 \mathrm{deg}$ ). This aftereffect, independent of the subject's movements, has been interpreted as a "normalization" effect, dealing essentially with perception. However, it has been shown to be stronger when the subject moves actively while wearing the rotating prism (Ebenholtz, 1966; Fiorentini, Ghez, \& Maffei, 1972). This "active" exposure can even lead to the full perceptual compensation of a 20-deg tilt exposure (Mikaelian \& Held, 1964).

The present experiments investigated whether visuomotor adaptation also would occur under conditions of a tilted visual feedback of one's own movements. For this purpose, we devised a situation in which the subject experiences visually his hand movements as "rotated" by a given angle with respect to their actual trajectory while the visual frame appears unchanged (normally oriented). In such a situation, no Gibson normalization effect or Wertheimer effect can be present, and the motor aftereffects that we observed should be related to a visuomotor shift rather than to a purely visual transform.

This work was supported by INSERM (Paris). Dr. Tzavaras's present address is Unité de Recherches Neuropsychologiques, (U 111 INSERM), 2 ter, rue d'Alesia, 75014 Paris, France. We thank Professor R. Held, Department of Psychology. Massachusetts Institute of Technology, Cambridge, for kindly reviewing our manuscript.

\section{METHODS}

The experiment required that the trajectory of hand movements be recorded during the tracking of lines. A technique has been developed that allows continuous recording of the contact of a hand-held stylus with an isotropically resistant surface (Bauer, Woods, \& Held, 1969; see full description in Prablanc \& Jeannerod, 1973). The output of this system was displayed on the screen of a storage oscilloscope and reflected in a mirror. In the normal closed-loop condition, the experimental arrangement was such that the subject, by looking through the mirror, could see the image of the oscilloscope spot in coincidence with the actual position of the tip of his stylus (Figure 1A). The position of the spot as seen through the mirror thus represented the reafferent signal from the position of the hand on the writing surface disposed horizontally. The situation was exactly the same as though the subject were writing in the dark with luminous ink. This experimental arrangement could be modified in several ways: (1) Rotation of the writing surface around its center resulted in tilting the visual feedback from displacement of the hand. In this condition, a line drawn by the subject at a given orientation appeared tilted in the mirror (rotation condition). (2) Turning off the brightness of the oscilloscope spot resulted in opening the eye-hand loop. The displacements of the stylus on the writing surface no longer produced visual reafferences (open-loop condition).

The experiment involved the usual three steps (Held $\&$ Gottlieb, 1958): preexposure test, in the open-loop + no-rotation condition; exposure, in the closed-loop + rotation condition (i.e., with an error-corrective feedback); postexposure test, in the open-loop + no-rotation condition.

During the experiment, the subject sat in front of the apparatus. Head movements were restrained by $a$ chin and forehead rest. The left eye was occluded, and the right eye viewed the mirror placed horizontally at $25 \mathrm{~cm}$ from the subject. The right hand, holding the stylus, was positioned below the mirror on the horizontal writing surface. limited by a circular frame. Total extent of hand movements was $13 \mathrm{~cm}$, which corresponded by reflection in the mirror to a maximum deviation of the oscilloscope spot of $13 \mathrm{deg}$ of visual angle. Procedure of the preexposure and postexposure tests was achieved in the same way. While the subject closed his eyes, the test figures were displayed and stored on the oscilloscope screen by the experimenter by means of another writing surface. The test figures, composed of linear segments, were displayed with the orientation as in Figure 2. The subject's task consisted of redrawing, with the stylus, each test figure as it appeared in the mirror (Figure 1B). 

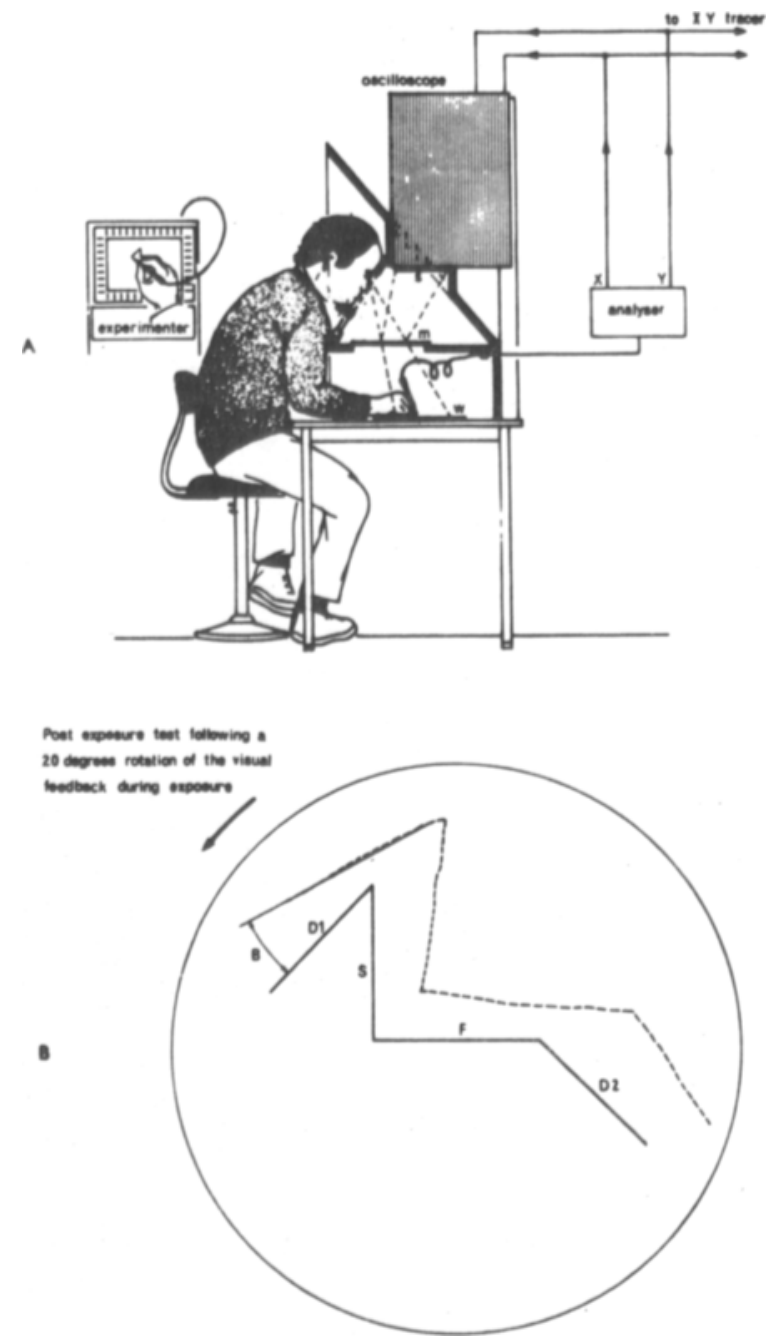

Figure 1. (A) Experimental arrangement: $s=$ screen of the oscilloscope, $m=$ mirror, $w=$ writing surface used by the subject. On the left is represented another writing surface used by the experimenter to display the test figures on the oscilloscope screen. (B) Example of a postexposure test: to the subject, - - - visuomotor response of the subject in the open-loop situation after a $20-\mathrm{deg} \mathrm{CCW}$ rotation of the visual feedback during exposure (arrow). The test figure and subject's response were both recorded on the $X Y$ tracer. $\hat{B}$ : angular deviation measured for orientation D1; F, S, D1, D2: frontal, sagittal, diagonal 1 , and diagonal 2 , respectively.

Data were recorded on an $X, Y$ tracer connected with the output of the stylus and were processed in the following way: The angular error between the actual orientation (as seen through the mirror) of a given test figure and its orientation as drawn by the subject was evaluated for each segment. Values obtained for segments of the same orientation from different test figures were pooled. Finally, values for each orientation (i.e., frontal, F; sagittal, S; diagonal $\%$ D1; and diagonal $1, D 2$ ) were averaged over the subjects for each experimental group. In the preexposure situation, these mean values reflect the simple angular error made by subjects drawing in an open-loop condition $(\bar{m} \AA)$. In the postexposure situation, they reflect the deviation produced by exposure to the conflict $(\overrightarrow{\mathrm{m}} \hat{\mathrm{B}})$.

In order to establish a normative background, data obtained in the preexposure situation (by using Test Figure 2a) were pooled over the whole sample of subjects (40 male and female right-handed students). Values of $\overline{\mathrm{m}} \hat{\mathrm{A}}$ were not found to be significantly different from zero for Orientations F, S, D2 (standard t tests). However, diagonal D1 was drawn systematically tilted toward the sagittal ( $\mathrm{m}$ $=3.92 \mathrm{deg}, \mathrm{p}<.001$ ) (Table 1 ).

\section{RESULTS}

\section{Experiment 1}

Twenty right-handed subjects were used. Ten were exposed to a 20-deg clockwise $(\mathrm{CW})$ rotation, and 10 others to a counterclockwise (CCW) rotation of the same amplitude. During an 8-min exposure, subjects were requested to draw lines in concordance with the visual frontal, saggital, and both diagonal orientations in the horizontal plane. Using the storage capacity of the oscilloscope, the subject had a continuous corrective feedback of his whole movement. The screen was erased every $1 \mathrm{~min}$. In the postexposure situation, subjects had to redraw five test figures presented sequentially in the same order as in Figure 2, b through $\mathrm{f}$. All subjects exhibited a relatively larger aftereffect in all four orientations, and for both directions of rotation (except for $\mathrm{D} 2$ in the $\mathrm{CW}$ direction). The values listed in Table 2 represent the nean angular aftereffect (i.e., $\overline{\mathbf{m}} \hat{\mathbf{B}}-\overline{\mathbf{m}} \hat{A}$ ) for each group of 10 subjects.

The presence of an aftereffect in this experimental situation does not rule out that subjects might have "learned" motor patterns for tracing lines at given orientations. Experiments 2 and 3 were designed to obviate this possibility.

Table 1

Mean Angular Errors ( $\overline{\mathrm{m}} \hat{\mathrm{A}}$, Degrees of Arc) in Pre-Exposure Testing 40 Subjects

\begin{tabular}{cccc}
\hline & \multicolumn{3}{c}{ Orientation } \\
\hline $\mathrm{F}$ & $\mathrm{S}$ & $\mathrm{D} 1$ & $\mathrm{D} 2$ \\
\hline+0.94 & +1.35 & +3.92 & -0.63 \\
n.s. & n.s. & .001 & n.s. \\
\hline
\end{tabular}

Note-Difference from zero tested by standard $t$ test.

Table 2

Mean Angular Aftereffects $(\overline{\mathbf{m}} \hat{\mathbf{B}}-\overline{\mathrm{m}} \hat{\mathbf{A}}$, Degrees of Arc) Observed in Experiment I

\begin{tabular}{lcccc}
\hline & \multicolumn{5}{c}{ Orientation } \\
\cline { 2 - 5 } $\begin{array}{c}\text { Direction } \\
\text { of Rotation }\end{array}$ & $\mathrm{F}$ & $\mathrm{S}$ & $\mathrm{D} 1$ & $\mathrm{D} 2$ \\
\hline $\mathrm{CW}$ & 4.2 & 12.4 & 10.4 & 3.6 \\
$\mathrm{~N}=10$ & .1 & .05 & .05 & n.s. \\
$\mathrm{CCW}$ & 7.9 & 6.9 & 7 & 6.8 \\
$\mathrm{~N}=10$ & .005 & .005 & .01 & .025 \\
\hline
\end{tabular}

Note-Difference from zero is tested by $t^{\prime}$ test using the Cochran and Cox approximation: Upper significance limits are given below each value. 


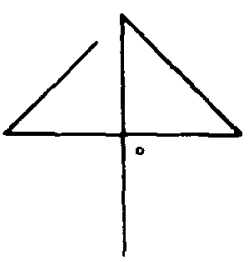

1

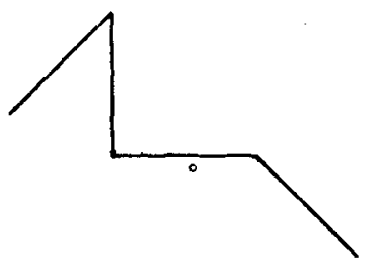

1

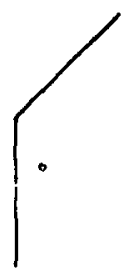

1

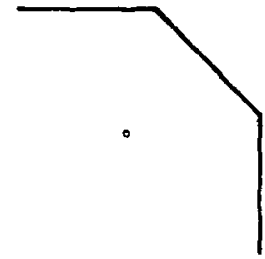

(

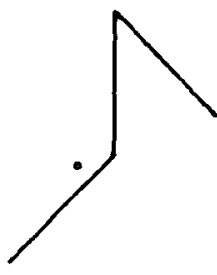

3

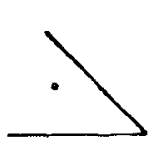

1

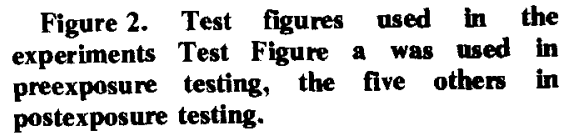

Figure 2. Test figures used in the experiments Test Figure a was used in postexposure testing.

\section{Experiment 2}

Ten subjects were used. Five were exposed to a $20-\mathrm{deg} \mathrm{CW}$ rotation, and five others to a $20-\mathrm{deg} \mathrm{CCW}$ rotation. Exposure time was the same as in Experiment 1. During exposure, the subjects were requested only to draw lines in the two visual diagonal orientations. Postexposure testing involved the same test figures as in Experiment 1, which allowed evaluating the aftereffect for both "exposed" (D1, D2) and "nonexposed" (F, S) orientations. The values listed in Table 3 show that an adaptive shift is present in all four orientations and for both conditions of rotation (see also Figure 3).

\section{Experiment 3}

This experiment, which also involved 10 subjects, was conducted in the same way as Experiment 2. However, during exposure, the subjects were requested only to draw lines in the visual frontal and sagittal orientations. Again, an adaptive shift was found also for "nonexposed" orientations (D1, D2) (Table 4; see also Figure 3). Direction of rotation, however, was an important variable in this experiment, since the aftereffect for D1 appeared significant only in the $\mathrm{CW}$ rotation condition, and for $\mathrm{D} 2$ only in the $\mathrm{CCW}$ rotation condition. Transfer of "adaptation" in this case seems to be limited to the diagonal orientation that is shifted toward the visual frontal axis.

\section{DISCUSSION}

Transfer of adaptation from exposed to nonexposed orientations (and thus not trained under visual control) seems to rule out simple motor "learning" as an explanation for the aftereffects that we have observed. However, the fact that we used line drawings as tests might have induced a sequential dependence between segments of different orientation in the same test figure. For instance, the ability to draw angles of 45,90 , and $135 \mathrm{deg}$ accurately might depend on previously learned strategies that were relatively uninfluenced by our experimental situation. If this were the case, a correct (adaptive) tracing of the first segment could result in drawing the whole test figure in the adaptive way. This possibility is disproved, however, by the absence of such a sequential dependence at the statistical level. Even though all segments of a given test figure were

Table 3

Mean Angular Aftereffects (Degrees of Arc) Observed in Experiment II

\begin{tabular}{ccccc}
\hline & \multicolumn{5}{c}{ Orientation } \\
\cline { 2 - 5 } $\begin{array}{c}\text { Direction } \\
\text { of Rotation }\end{array}$ & $\mathrm{F}$ & $\mathrm{S}$ & $\mathrm{D} 1$ & $\mathrm{D} 2$ \\
\hline $\mathrm{CW}$ & 8.2 & 4.3 & 15.4 & 6.1 \\
$\mathrm{~N}=5$ & .0005 & .05 & .0005 & .005 \\
$\mathrm{CCW}$ & 13.7 & 7.5 & 7.9 & 6.8 \\
$\mathrm{~N}=5$ & .0005 & .005 & .01 & .05 \\
\hline
\end{tabular}

Note-Difference from zero is tested by $t^{*}$ test using the Cochran and Cox approximation: Upper significance limits are given below each value.

Table 4

Mean Angular Aftereffects (Degrees of Arc) Observed in Experiment III

\begin{tabular}{ccccc}
\hline & \multicolumn{5}{c}{ Orientation } \\
\cline { 2 - 5 } $\begin{array}{c}\text { Direction } \\
\text { of Rotation }\end{array}$ & $\mathrm{F}$ & $\mathrm{S}$ & $\mathrm{D} 1$ & $\mathrm{D} 2$ \\
\hline $\mathrm{CW}$ & 10.9 & 10 & 16.2 & 8.5 \\
$\mathrm{~N}=5$ & .005 & .005 & .0005 & .1 \\
$\mathrm{CCW}$ & 14.1 & 21.4 & 3.3 & 18.5 \\
$\mathrm{~N}=5$ & .0005 & .0005 & n.s. & .005 \\
\hline
\end{tabular}

Note-Diffelence from zero is tested by $t^{\prime}$ test using the Cochran and Cox approximation: Upper significance limits are given below each value. 


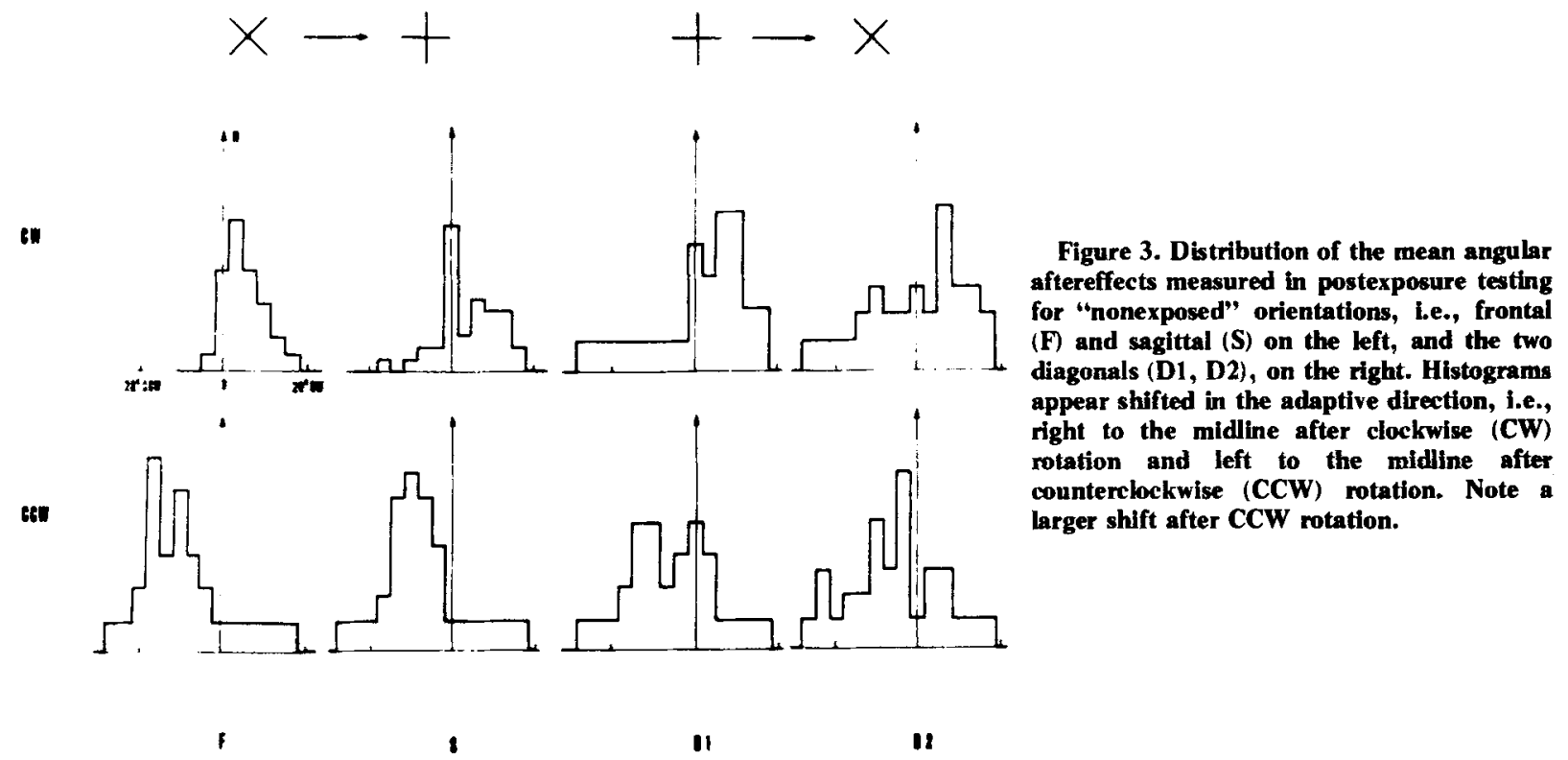

"adapted." the amount of the adaptive shift could vary from one segment to the next. No correlation was found between the value of adaptive shift for each segment and the corresponding value for the segment that comes next in the drawing (Bravais-Pearson test, $r=.35$, nonsignificant).

Generalization of the adaptive shift from two perpendicular orientations to the whole set of coordinates implies a "recalibration" of the mechanism that generates motor outputs in relation to the retinal orientation of the stimulus. Such a generalization hypothesis leads to the prediction that the perception of other spatial coordinates should also be moditied by adaptation to a rotated visuomotor feedback. For instance, the set of egocentric references used in the control of motor programs in the absence of visual guidance might be perceived as "rotated." Experiments in progress show that this is indeed the case. After the same adaptation procedure as described here, the subjective sagittal body axis seems to be "displaced." When asked, with their eyes closed and with the adapted arm. to draw on the horizontal writing surface a line directed toward their vertical body axis, all subjects show a significant mislocation in the "adaptive" direction (Prablanc, Jeannerod, and Tzavaras, in preparation). This mislocation resembles the errors in pointing "straight ahead" observed by several authors after adaptation to laterally displacing prisms (Harris, 1963; Bauer \& Efstathiou, Note 1).

\section{REFERENCES}

Bauer, J. A.. Woods, G. D.. \& Held, R. A device for rapid recording of positioning responses in two dimensions. Behavior
Research Methods \& Instrumentation, 1969, 1. 157-159.

EBenholtz. S. M. Adaptation 10 a rotated visual field as a function of degree of optical tilt and exposure time. Joumal of Experimental Psychology. 1966, 72. 629.634.

Fiorentini, A., Ghez, C., \& Maffei, L. Physiological correlates of adaptation to a rotated visual field. Journal of Physiology, 1972, 217, 313-322.

Gibson. J. J. Adaptation after effect. and contrast in the perception of tilted lines. Joumal of Experimental Psychology, 1937. 20, 553-569.

Harris, C. S. Adaptation to displaced vision: Visual, motor or proprioceptive change? Science. 1963, 140, 812-813.

Held. R., \& Freedman, S. Plasticity in human sensorimotor control. Science, 1963. 142, 455-462.

Held. R., \& Gotrlieb, N. Technique for studying adaptation to disarranged hand eye coordination. Perceptual and Motor Skills. 1958. 8. 83-86.

Mikaelian, H., \& Held, R. Two types of adaptation to an optically rotated visual tield. American Joumal of Psychology, 1964. 77, 257-263.

Prabl.anc, C.. \& Jeannerod, M. Continuous recording of hand position in the study of complex visuo-motor tasks. Neuropsychologis, 1973, 11, 123-125.

\section{REFERENCE NOTE}

1. Bauer, J., \& Efstathiou. E. Effects of adaptation to visual displacement on pointing "straight ahead." Paper read at Eastern Psychological Association, 1965.

\section{NOTE}

1. Only errors in orientation were measured. Possible errors in position of the whole test figure, as compared to its actual position in the mirror, were not taken into account. Errors of this type were present in most subjects, as commonly observed in experiments where the subject has to point at targets (e.g. Held \& Freedman, $19(3)$ ).

(Received for publication February 27, 1974; revision received December 6.1974.$)$ 